\title{
Ordenamiento de los registros semióticos en la didáctica del álgebra en la escuela secundaria. Correspondencia con la enseñanza de expresiones algebraicas racionales ${ }^{1}$
}

\section{Ordination of semiotic records in the algebra didactics at the secondary school. Correspondence with the teaching of rational algebraic expressions}

Eugenio Valiero

Melissa Barrionuevo ${ }^{3}$

Flavia Villenas ${ }^{4}$

\begin{abstract}
Resumen: La presente investigación fue realizada en el contexto de Formación Docente Inicial (de un Instituto Superior Estatal de la Provincia de Entre Ríos, Argentina), para conocer la utilización de registros semióticos en experiencias reales de enseñanza, en la observación de los ordenamientos representacionales que realiza un profesor en sus propuestas didácticas. Se tomó como base conceptual la Teoría de Registros de Representación Semiótica, de Raymond Duval. ${ }^{5}$ La caracterización cualitativa comprendió un estudio de casos mediante entrevistas y observaciones de clases, en una escuela urbana de la ciudad de Gualeguay. Se analizó el modo en que se ordenan los registros en la enseñanza de expresiones algebraicas racionales en $4^{\circ}$ año de la Educación Secundaria y sus posibles errores didácticos, desde una concepción social,
\end{abstract}

Fecha de recepción: 27 de agosto de 2017. Fecha de aceptación: 08 de febrero de 2021.

1 Una Expresión Algebraica Racional es la razón, cociente o fracción entre polinomios.

2 Instituto de Enseñanza Superior Dr. Luis Federico Leloir, Profesorado de Educación Secundaria en Matemática, , eugeniovaliero@gmail.com, orcid.org/0000-0001-9392-0882

3 Instituto de Enseñanza Superior "Dr. Luis Federico Leloir", melissabarriouevo@hotmail.es, orcid. org/0000-0001-7428-688X

4 Instituto de Enseñanza Superior "Dr. Luis Federico Leloir", flaviavictoriavillenas@hotmail.com.ar, orcid. org/0000-0003-2013-5140

5 Profesor francés de la Universidad del Litoral dedicado a estudios acerca de los procesos cognitivos en enseñanza y en aprendizaje de la Matemática. 
educativa, y didáctica. Se consideró que lo representacional podría constituirse en una condición secuencial cuyo impacto se liga a los procesos de abordaje de la Didáctica Específica de la Matemática Escolar. Se partió del supuesto de que toda situación de enseñanza conlleva formatos necesarios de comunicación, y formulación de dispositivos representacionales visuales, auditivos, verbales, escritos.

Palabras clave: Teoría de los Registros Semióticos, matemática, enseñanza del álgebra, expresiones algebraicas racionales, didáctica de la matemática.

\begin{abstract}
The present investigation was carried out in the context of a Teacher Training College (State Superior Institution in the Province of Entre Ríos, Argentina) to know the use of semiotic registers in real teaching experiences. The study is based on the observation of the representative arrangements that a teacher makes in his proposal didactics. Raymond Duval's Theory of Records of Semiotic Representation was taken as a conceptual base and the characterization included a qualitative cases study through interviews and direct observations of classes in an urban school at the city of Gualeguay. In this research the way in which the different registers are ordered in the teaching of rational algebraic expressions in the 4th year at Secondary Education was analyzed to identify programming aspects of the practical proposals formulated by teachers that could lead to didactic error. The research question was approached from a social educational and didactic conception. It was considered that it could constitute an important sequential condition whose impact is linked to the processes of approaching Specific Didactics of School Mathematics content since it was assumed that every teaching situation involves necessary formats for communication and formulation of visual, auditory, verbal, written devices with representative attributes.
\end{abstract}

Keywords: Theory of Semiotic Records, math, algebra teaching, rational algebraic expressions, mathematics teaching. 


\section{INTRODUCCIÓN}

Desde principios de 1970, las investigaciones en Enseñanza de la Matemática pretenden conocer básicamente tres aspectos: dificultades de comprensión, modos de resolver situaciones problemáticas, y funcionamiento cognitivo en la actividad matemática escolar. Existen, en ese sentido, diferentes enfoques de la problemática didáctica como lo es el aspecto representacional.

A efectos del presente estudio, se toma como referencia la llamada Teoría de los Registros de Representación Semiótica (TRRS), de Raymond Duval, según la cual la construcción conceptual y de comunicación, en Matemática, dependen de: la actividad de formación de representaciones (tablas, gráficos, notaciones, lenguaje verbal), y de procesos de transformación denominados tratamiento, y conversión, de los sistemas de signos (registros), que constituyen dichas representaciones (Duval, 1999).

La actividad de sustitución de objetos matemáticos escolares por representaciones semióticas depende de las transformaciones y de las relaciones entre signos asociados de manera compleja mediante reglas. Esta interviene en la comunicación, y en nuevos conocimientos. Aceptando la existencia de diferentes sistemas de representación (mental, icónica, material, semiótica, etc.), se considera registro ${ }^{6}$ solo a los sistemas semióticos que permiten las transformaciones de representaciones antes mencionadas (Duval y Sáenz-Ludlow, 2016). El lenguaje verbal natural, las representaciones gráficas y tabulares, las expresiones numéricas, las notaciones algebraicas, y los sistemas icónicos, conforman registros semióticos (RS).

Los errores o dificultades de aprendizaje en matemática se asocian principalmente a inconvenientes de conversión, es decir, a transformaciones representacionales desde un tipo de registro dado, a otro, por ejemplo, una ecuación de lenguaje algebraico a gráfico cartesiana (Duval y Sáenz-Ludlow, 2016). Sara et al. (2006), presentaron un análisis de los errores de representación semiótica cometidos por los estudiantes en los exámenes parciales de Álgebra Lineal y Geometría Analítica, en carreras tecnológicas de ingeniería. Observaron las dificultades para resolver tres tipos de problemas:

6 Verbal o lingüístico (RRVL), numérico (RRN), algebraico (RRA), simbólico (RRS), geométrico (RRGe), gráfico (RRGr), computacional (RRC), tabular (RRT). 
(...) 1을 dadas las ecuaciones paramétricas de una recta L, hallar la proyección de un punto A de coordenadas conocidas (registro tabular), sobre L; (...) 2º) dada la ecuación vectorial paramétrica de una recta, definir $L$ como intersección de dos planos; (...) 3ํ) analizar la validez de la expresión que afirma que si dos vectores conforman un conjunto linealmente independiente en un espacio vectorial de dimensión mayor que dos, entonces el conjunto de dos combinaciones lineales de esos vectores con coeficientes determinados, salvo uno, también es linealmente independiente cualquiera sea el coeficiente indeterminado (Sara et al., 2006, pp. 321-322).

Encontraron que los errores, se debían a dificultades de aplicación, a confusión entre representación y objeto matemático, a errores de tratamiento, a fallas lógicas, y a déficit de definición y construcción conceptual; y atribuyeron los mismos a un insuficiente trabajo con diferentes registros de representación (Sara et al., 2006). Otros autores han tratado el tema de los RS tomando como objeto de análisis la intervención de un número significativo de ellos tanto en la tarea docente como en la propia actividad de aprendizaje. Sugirieron fomentar instancias de creación de nuevos signos y formatos de representación como actividad de acrecentamiento cognitivo (Oviedo et al., 2011).

Otra investigación analizó, mediante el método de entrevista, la relación entre la coordinación de representaciones en la acción de resolver situaciones diversas de transformaciones lineales con estudiantes de Licenciatura en Matemática, teniendo como referencia la TRRS (Ramírez Sandoval, et al., 2013).

Se partió de la premisa de que es posible producir un fracaso en el aprendizaje, en caso de no producirse una adecuada coordinación, y concluyó que, si bien estos procesos favorecen la obtención satisfactoria de soluciones, no la garantizan (Ramírez Sandoval et al., 2013).

La temática de la coordinación de los RS acerca de la intencionalidad estructural de los autores de diferentes libros universitarios en la propuesta de formación trigonométrica, y los modos de representación semiótica en la secuenciación de contenidos matemáticos, reconoció en su estudio, la existencia de propuestas bibliográficas a las que se denominó monorregistro de las funciones trigonométricas, debido a que:

(...) aunque la conversión de unidades, la definiciones de las funciones trigonométricas y las propiedades de acotamiento y periodicidad se presentan en tres registros de representación diferentes (lengua natural, algebraico y gráfico), no hay conversión de unidades entre al menos dos de estos registros; antes bien, todo el 
tratamiento se da solo en la lengua natural y los otros dos registros sirven de apoyo: el algebraico para dotar de una ecuación y gráfico de auxiliar a lo dicho en lengua natural (Aponte Rodríguez, 2016, p. 82).

Trabajos vinculados a enseñanza de funciones racionales en $5^{\circ}$ año de Educación Secundaria (desde la perspectiva de las denominadas Teoría Antropológica de lo Didáctico, Teoría de Juego, y Teoría de Campos Conceptuales), pusieron en discusión alcances y limitaciones en actividad de estudio e investigación (AEI) a partir de la pregunta ¿cómo operar con curvas cualesquiera si solo se dispone de la representación gráfica de la misma y de la unidad en los ejes? Utilizando una metodología cualitativa, etnográfica y exploratoria, se propuso describir y justificar si la AEI permitiría construir las propiedades fundamentales de funciones racionales de manera significativa. Se observaron clases en dos cursos de una misma escuela donde la actividad propuesta consistió en buscar la gráfica más razonable de una función racional conociendo la gráfica del polinomio resultado de la división de los polinomios de la función en cuestión. Obtuvieron que es posible la construcción de propuestas didácticas mediante AEl, y la recuperación del sentido conceptual, aunque ello se lograra de manera imperfecta (Gazzola et al., 2011).

La presente investigación abordó la forma en que el ordenamiento de distintas representaciones semiótico-didácticas, se establece en jerarquías de secuenciación de los registros en la enseñanza de expresiones algebraicas racionales. El objetivo de estudio se centró en conocer aspectos cualitativos del orden en que son presentados por los profesores de educación secundaria, y el modo en que se podrían generar errores didácticos. La investigación descriptiva se encuadró dentro del área de Educación; y consistió en la realización de observaciones de clases, mediante una metodología del tipo cualitativa, y entrevistas a dos docentes de Matemática, de una escuela secundaria urbana, de la ciudad de Gualeguay (Argentina). 


\section{MARCO TEÓRICO}

\subsection{ERRORES EN ENSEÑANZA Y EN APRENDIZAJE}

Los errores del aprendizaje "constituyen la manifestación exterior de un proceso complejo donde se ponen en juego muchas variables: docente, estudiante, currículo, contexto sociocultural, entre otros" (Engler et al., 2015, p. 26). Por ello no es adecuado pensarlos únicamente como fracasos del aprendizaje, y deben detectarse de inmediato para ser superados. Rico (1997), admite que los errores pueden ser de origen ontogénico, didáctico, semiótico, cultural y epistemológico, entre otras clasificaciones.

Si bien interesa centrar la mirada en los ordenamientos registrales, se consideraron solamente los denominados errores didácticos, y se los distingue, en su análisis, de los de origen epistemológico debido a que, habitualmente, son pensados indistintamente en las prácticas de enseñanza y, además, porque la TRRS hace referencia directa a aspectos de adquisición conceptual, lo cual se vincula más a estos últimos.

Popper, Lakatos y Bachelard (s.f.), citados por Rico (1997, p. 6), realizan aportes al esclarecimiento de este último tipo. Así, desde la perspectiva epistemológica, ${ }^{7}$ se consideran a los errores como conocimientos deficientes e incompletos según el contexto cognitivo, que imposibilitan la permanente adquisición y consolidación de nuevos conocimientos; forman parte de la construcción del mismo, y surgen en un marco conceptual consistente, basado en conocimientos adquiridos previamente, es decir, cuyas limitaciones están relacionadas con el propio significado de los conceptos matemáticos.

Es posible diferenciar dos tipos de profesores los cuales tienen visiones distintas acerca de los errores del aprendizaje: el profesor convencional-tradicionalista, y el profesor moderno-avanzado (Rico, 1997). Los primeros, consideran a los errores como un dato objetivo de desconocimiento de un estudiante que debe ser corregido, penalizado. Sostienen que este tratamiento del error prepara a los estudiantes para la vida y para la inserción social; cometer un error representa una conducta inadecuada que hay que superar. Los segundos, en cambio, ven en los errores una muestra de un conocimiento construido

Conjunto de convicciones, de conocimientos y de saberes científicos, que tienden a decir cuáles son los conocimientos de los individuos o grupos de personas, funcionamiento, formas de establecer su validez, de adquirirlas y por tanto enseñarlas y de aprenderlas. (D'Amore, 2004) 
parcialmente, resultado de un proceso en curso a cuyo progreso el educador debe contribuir, procurar una revisión para mejorar el significado erróneo. Así, se debería partir de una reorganización de los conocimientos anteriores en nuevas situaciones con el objetivo de obtener resultados positivos y conseguir que los estudiantes logren un aprendizaje significativo (Rico, 1997).

\subsection{ERROR, REPRESENTACIÓN SEMIÓTICA, Y APRENDIZAJE}

Respecto de la dependencia del pensamiento matemático, del lenguaje, y de otros sistemas de representación, algunos autores afirman la autonomía de la conceptualización con relación a toda actividad semiótica. ${ }^{8}$ Sin embargo, estas cuestiones están en el centro de las dificultades ${ }^{9}$ que los alumnos encuentran en su aprendizaje (Duval, 2006).

En Matemática tales dificultades con frecuencia se interpretan como errores, y afectan a la construcción del conocimiento matemático, su tratamiento, comprensión y valoración. Para Duval (2004), esas construcciones se dan por representaciones semióticas, es decir las que se constituyen por uso de signos ya sea en lenguaje formal, fórmulas algebraicas o figuras geométricas, por ejemplo, y serían el medio por el cual se exteriorizan las representaciones mentales y mediante las cuales se hacen visibles o accesibles.

Las investigaciones referidas a coordinación dan cuenta de cierta dificultad de los estudiantes en adquirir la habilidad para cambiar de registro de representación. Duval (2006) señala que:

- Los contextos de representación utilizados en matemática son semióticos.

- El procesamiento matemático implica siempre una transformación de representaciones.

- Se requiere una coordinación interna para elegir una representación según el propósito de la actividad.

8 Adquisición de una representación realizada por signos.

9 Cuando se mencionan las dificultades, en el contexto en que aquí se las concibe, se hace referencia al aprendizaje (actividad práctica y cognitiva del alumno), conocidas como obstáculos epistemológicos; en el sentido amplio Bachelard (2000) los asocia al de contrapensamientos (Bacherlard, 2000). No se harán en adelante distinciones con la idea de error. 
Para comprender los obstáculos de aprendizaje con lo que ello se vincula se deben distinguir dos clases de transformaciones: la conversión en la que se cambia el sistema semiótico sin cambiar los objetos indicados; por ejemplo, se puede adoptar una representación del objeto matemático ${ }^{10}$ "la mitad de un entero" (lengua natural del registro verbal o lingüístico), y convertir mediante la fracción 11/2 (registro numérico); y el tratamiento, en donde se opera manteniendo el mismo registro, por ejemplo, continuando con la expresión numérica anterior, transformar en el mismo registro a la expresión decimal 0,5. (figura 1).

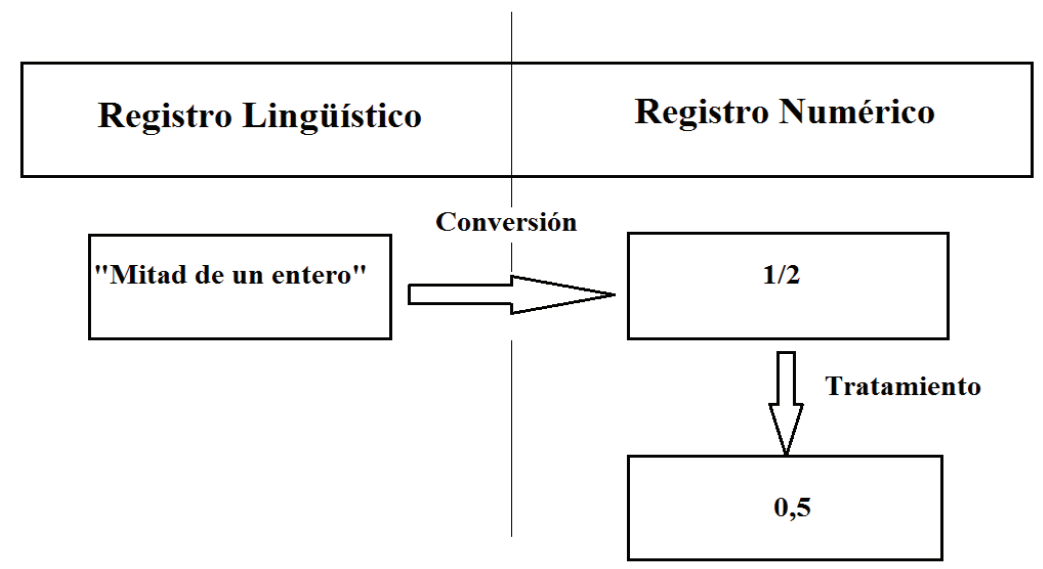

Figura 1. Ejemplo de transformaciones semióticas en Matemática.

Muchas veces estas transformaciones se pueden identificar claramente, o se dan en etapas de un proceso, o en un contexto, por ejemplo, en un registro, en resolución de problemas; también, hay situaciones en las que se requiere movilizar dos o más registros. En este sentido la conversión y el tratamiento podrían ser considerados como un todo, un entorno metodológico y estratégico en la actividad matemática de resolver problemas, o en el trabajo algebraico; sin embargo, la conversión es un proceso cognitivo más complejo que el tratamiento, y puede considerarse como el origen de la comprensión.

\footnotetext{
${ }^{10}$ Se concibe a los objetos matemáticos en su constitución abstracta la cual haya adquirido identidad operativa o conceptual: un objeto, como entidad, es una cosa a la cual nos referimos, o de la que podemos hablar, sea en condición real, imaginaria o de otro tipo, y su existencia depende de los significados que le asignan ciertos grupos sociales (Blumer, 1982).
} 


\subsection{REPRESENTACIÓN SEMIÓTICA, DIDÁCTICA, Y ERROR}

La Enseñanza de la Matemática debe satisfacer dos requisitos que en un punto entran en conflicto: por un lado, todo concepto matemático se sirve de representaciones, dado que no se dispone de objetos para exhibir en su lugar por lo que la conceptualización ${ }^{11}$ debe necesariamente pasar a través de registros representativos. Y, por otro lado, los objetos matemáticos representados nunca deben confundirse con el contenido de las representaciones utilizadas (Duval, 2006).

Un objeto matemático, según Godino (2002), también se concibe como "todo lo que es indicado, señalado, nombrado cuando se construye, se comunica, o se aprende matemáticas" (Godino, 2002, como se citó en D'Amore y Godino, 2007, p. 208).

Duval (1996) citado por D'Amore (2009), sostiene que no existe concepto sin sistema de signos:

Todas las funciones psíquicas superiores se hallan unidas por una característica común superior, la de ser procesos mediados, es decir el incluir en su estructura, como parte central y esencial del proceso en su conjunto, el empleo del signo como medio fundamental de orientación y de dominio de los procesos psíquicos... La lista central del proceso de formación de los conceptos es el uso funcional del signo, o de la palabra, como medio que permite al adolescente de someter a su poder las propias operaciones psíquicas, de dominar el curso de sus propios procesos psíquicos (...) (Duval 1996, como se citó en D'Amore, 2009, párr. 11).

En Matemática, los sistemas semióticos son principalmente utilizados para operar (tratamiento), por eso es importante reconocer que el contenido de cada representación depende de los conceptos u objetos representados y del registro utilizado. Cambiar de un sistema a otro significa cambiar el contenido de la representación sin cambiar las propiedades matemáticas representadas. Por ello muchos estudiantes se ven atrapados en esta función semiótica, que conlleva a la falta de comprensión.

El primer paso para el entendimiento es la posibilidad de transferir lo que se ha aprendido a nuevos y diferentes contextos semióticos, por lo que una de las condiciones es que haya una coordinación interna entre los diversos sistemas

\footnotetext{
${ }^{11}$ La conceptualización es el pasaje de los conceptos-como-instrumento a los conceptos-como-objeto y una operación lingüística esencial en esta transformación es la nominalización, es una apropiación consciente.
} 
de representación disponibles para usar. Se dice entonces que se establece una función semiótica entre dos objetos matemáticos cuando entre dichos objetos se establece una dependencia representacional o instrumental, "esto es, uno de ellos se pone en el lugar del otro o uno es usado por otro" (D'Amore y Godino, 2007, p. 210).

Los profesores deben ser capaces de relacionar de muchas maneras representativas los contenidos, en distintas situaciones, para que así los alumnos puedan desempeñar un rol activo, como describen las teorías constructivistas; tienen un papel central; proporcionar estas situaciones, mediante resolución de problemas, debates para involucrar a los estudiantes en la resolución, aclaración de ideas, conceptos y terminologías. Debe ser activo y creativo y debe respetar las individualidades de los alumnos y no perder de vista que un concepto matemático tiene diferentes niveles de abstracción y generalidad.

Si se abordara un concepto con un significado no apropiado para un problema dado, o que no sea semióticamente diseñado, o representado en forma adecuada, la propuesta didáctica funcionaría como un obstáculo. Al respecto, debe considerarse que los errores didácticos introducen formas de pensar y conceptualizar limitadas por maneras de enseñar. Sierpinska (1992), al referirse a los obstáculos señala: "No obstante, se debe prestar especial atención, a aqueIlos que no son simples resultados de formas particulares de su enseñanza, ni idiosincrásicos, ni algo que ocurre a una persona o dos, sino que está más extendido... (...)" (Sierpinska 1992, como se citó en Bohorquez et al., 2009, p. 480).

Brousseau (1983), caracteriza al obstáculo didáctico como resultado de una opción o de un proyecto del sistema educativo, de las elecciones que se hacen para establecer la situación de enseñanza. Destaca, además, que es siempre el fruto de la interacción del alumno con un medio, y, en efecto, el error (epistemológico) es una manifestación de dicho obstáculo, es decir la consecuencia causal cuando los errores son persistentes y reproducibles:

Los trabajos conformes a las concepciones de Bachelard y de Piaget muestran también que el error y el fracaso no tienen el rol simplificado que en ocasiones uno quiere hacerles jugar. El error no es solamente el efecto de la ignorancia, de la incertidumbre, del azar que uno cree en las teorías empiristas-conductistas del aprendizaje, sino el efecto de un conocimiento anterior, que tenía su interés, su éxito, pero que, ahora, se revela falso, o simplemente inadaptado. Los errores de este tipo no son erráticos e imprevisibles, se han constituido en obstáculos. Tanto en el 
funcionamiento del maestro como en el del alumno, el error es constitutivo del sentido del conocimiento adquirido (Brousseau, 1983, p. 67).

También puede vincularse el error de origen epistemológico, al error didáctico que aquí se intenta describir.

\subsection{TRANSPOSICIÓN DIDÁCTICA Y CONTEXTO SEMIÓTICO}

Un contenido, para ser enseñado, sufre transformaciones cuya finalidad es la de hacerlo apto para ocupar un lugar entre los objetos de enseñanza. También adquiere diferentes formatos semióticos. Chevallard denomina al saber científiCo, al ser transformado en saber posible de ser enseñado, con el nombre Transposición Didáctica (Chevallard, 1993).

Es preciso que exista dicha transposición, ya que en innumerables casos la distancia didáctico-pedagógica o epistémica entre el objeto de saber y el objeto de enseñanza, es inmensa. Sin embargo, no se debe confundir conversión de un registro semiótico a otro con la efectiva transposición didáctica que se realiza a todo objeto de saber escolar: "Un objeto de saber solo llega a la existencia como tal, en el campo de conciencia de los agentes del sistema de enseñanza, cuando su inserción en el sistema de los objetos a enseñar se presenta como útil para la economía del sistema didáctico" (Chevallard, 1993, p. 57).

Los profesionales de la educación deben tener en cuenta distintas estrategias didácticas para prevenir (o superar) errores de origen didáctico en función de dicha economía, mediante las transposiciones didácticas más adecuadas, sin confundirlas con los posibles errores epistemológicos o con conversiones semióticas deficientes. Al respecto Duval (2017), destaca la importancia de los procesos cognitivos y de su estrecha relación con los RS. La actividad matemática requiere un trabajo docente que enfatice en la enseñanza de las conversiones registrales, las cuales no resultan en los aprendizajes, ni intuitivas ni espontáneas: "la conversión de las representaciones semióticas constituye la actividad cognitiva menos espontánea y más difícil de adquirir para la gran mayoría de los alumnos" (Duval, 2004, p. 49).

Por ello se sostiene que los sistemas de representación semiótica tanto en su tratamiento como en su conversión, están estrechamente vinculados con los sistemas didácticos, y principalmente en la intervención necesaria del registro lingüístico como nexo de los demás registros. 


\section{DESARROLLO DEL TRABAJO}

La presente investigación fue llevada a cabo en el contexto de formación de profesores de Matemática, tomando como campo de observación el ámbito de la enseñanza secundaria, en una escuela de la ciudad de Gualeguay, Provincia de Entre Ríos, Argentina. Se propuso analizar un caso de implementación de una propuesta de enseñanza real en aula. La misma se realizó simultáneamente en dos cursos de igual nivel formativo, correspondiente al $4^{\circ}$ año, de entre 15 y 16 años de edad, cuyos grupos de estudiantes compartían similitudes en las condiciones contextuales y académicas. La propuesta fue diseñada por las docentes de los respectivos cursos en acuerdos didácticos y curriculares también compartidos (ambas informantes fueron entrevistadas).

El plan tuvo como propósito el de responder dos preguntas de investigación que fueron ordenadas según la prioridad de los objetivos de análisis en:

- Pregunta primaria: ¿De qué manera se ordenan los registros de representación semiótica en la enseñanza de expresiones algebraicas racionales en una clase de Matemática de $4^{\circ}$ año de educación secundaria en una escuela de la ciudad de Gualeguay?

- Pregunta secundaria: ¿Cómo se vinculan esos ordenamientos representacionales en el diseño de propuestas didácticas con la posibilidad de inducir a errores didácticos?

Se trató de una investigación de caso, de tipo cualitativa donde se consideró no relevante el número de clases a observar puesto que no se procuró obtener resultados generalizables y se optó por una metodología comparativa entre no más de dos situaciones de enseñanza del contenido curricular de interés que permitieran caracterizar y describir el caso didáctico (enseñanza de expresiones algebraicas racionales).

\subsection{ENTREVISTA A DOCENTES DE MATEMÁtICA}

Se entrevistó a dos docentes, de dos cursos de idéntica graduación escolar ( $4^{\circ}$ año de Educación Secundaria), en la misma época del año académico, previamente a la observación de sus clases. Para conocer la utilización de RS, su postura frente a los errores didácticos, y la manera de afrontar las dificultades 
de la enseñanza y del aprendizaje, se les formularon preguntas semi-estructuradas de carácter abierto (apartados 3.3 y 3.4).

\subsection{OBSERVACIONES DE CLASE DE MATEMÁtICA EN 4TO AÑO}

Luego de la entrevista, se realizó una observación de clase por cada docente, en los grupos de clases donde se desarrollarían los contenidos referidos a Expresiones Algebraicas Racionales, a fin de describir los RS utilizados. Se tomaron datos del proceso de enseñanza, las anotaciones de los estudiantes, sus preguntas e intervenciones en clase, las conceptualizaciones que ellos hacían, los obstáculos en las operaciones que realizaban para resolver las expresiones algebraicas racionales, y la modalidad de resolución de actividades y ejercicios del tema.

\subsection{ENTREVISTA NÚMERO 1}

De la primera entrevista surge que la docente utiliza como estrategia didáctica, la vinculación de los conceptos a enseñar con temas anteriormente vistos, es decir, la tarea de referir un nuevo contexto semiótico-representacional, a conocimientos registrales previos.

- ¿Qué estrategias didácticas consideras que colaboran al mejor entendimiento de las Expresiones Algebraicas Fraccionarias?

- (...) Yo lo voy relacionando siempre, más que nada, con las operaciones con fracciones comunes (RRN y transformación de tratamiento), simples digamos, para que ellos se vayan ubicando, comparando siempre y les resulta un poco más fácil de entender, porque si no es como que son temas totalmente desconectado (...).

Este tipo de estrategia, utilizada por la profesora, se ajusta al tipo de didáctica sugerida por Brousseau (1983), aludida anteriormente, ya que permite que el alumno emplee sus conocimientos previos frente a situaciones novedosas, a la vez que incluye a sus estructuras las nuevas explicaciones y los nuevos contextos de aplicación. Lo observado en la clase indica que no resultó eficaz ya que los alumnos no lograron vincular los conceptos nuevos con los anteriormente vistos. 
Cuando se le preguntó sobre los recursos y actividades propuestas para el tema, la profesora contestó que comienza con ejemplos en el pizarrón y luego ejercitación, para reforzar la utilización del registro algebraico principalmente, tanto por parte del docente como por parte de los alumnos.

- Hoy vamos a observar una clase sobre Expresiones Algebraicas Fraccionarias ¿De qué manera piensa desarrollar el tema? ¿Cómo iniciaría la clase?

- (...) Hay poca didáctica para este tema, comienzo dando un ejercicio de ejemplo (transformación de tratamiento), y después les propongo varios a ellos para que se familiaricen con el tema (...).

- ¿Qué actividades realizan los alumnos?

- (...) Fundamentalmente ejercicios de aplicación (...).

Se observó así, que la docente propone tratamientos dentro del mismo registro y no conversiones, como por ejemplo si se plantearan situaciones problemáticas en las que los estudiantes debieran transformar en otras formas semióticas las consignas dadas en RRVL.

Al preguntar sobre los errores habituales de los alumnos, respondió que los mismos no podían aplicar temas vistos anteriormente a nuevos contextos (por ejemplo, los casos de factoreo, simplificación de expresiones, o propiedad distributiva).

- ¿Cuáles son las dificultades más usuales que presentan los estudiantes en el momento de comprender las Expresiones Algebraicas Fraccionarias?

- Simplificar, aplicar los casos de factoreo, aplicar la propiedad distributiva.

- ¿Crees que estas se deban a la falta de conocimientos previos, o conocimientos incompletos del alumno? ¿Por qué crees que sucede?

- (...) No sé si no lo tienen, porque ellos me demuestran lo contrario en la evaluación, pero es como que ellos terminan el tema y cierran la cortina, es como que después van a ver algo totalmente distinto. No entienden que en Matemática está todo relacionado (...) Es tan difícil saberlo, ellos viven el día a día, los adolescentes viven el día a día, por eso yo les digo que vayan haciendo anotaciones, que se hagan su propia carpeta, entonces tienen que hacer las anotaciones que consideren importantes, pero para que ellos entiendan, no para que entienda yo y para que no se olviden, porque yo puedo asegurar que la próxima clase vengo a repasar y ya se olvidaron cómo es que se hacía (...). 
Se puede interpretar que la perspectiva de enseñanza prevé los errores o dificultades que se presentan en la clase sin potencialidad de producir, a partir de ellos, nuevas formas de enseñar, es decir de construcción de una secuencia registral de representación diferente, pues se vincularía al tipo denominado error convencional.

Por último, al preguntar cómo trabaja sobre esas dificultades, señaló que aplica distintas técnicas, por ejemplo, repasar lo dado antes de la evaluación, dar ejercicios de aplicación más sencillos (transformación de tratamiento), o explicar el tema nuevamente ya que no se puede avanzar si los alumnos no han comprendido.

- ¿Cómo hace usted para afrontar esas dificultades?

- (...) generalmente hago un repaso rápido, por ejemplo, antes de la evaluación, doy un módulo de repaso y en los otros cuarenta minutos siguientes les tomo la evaluación, generalmente hago eso para que no fracasen. El que no estudió, al que no le importa, fracasa igual, pero al menos así puedo salvar un alto porcentaje (...).

- ¿Haces modificaciones en la planificación?

- Siempre surgen cosas que te obligan a modificar lo que planificaste, o porque no entendieron o porque faltan, por ahí te salen unas clases perfectas.

- ¿Trabajas sobre las correcciones?

- Lamentablemente hay que volver a empezar, repasar, no puedo dar algo por sabido porque así no es posible avanzar (...).

Lo observado se correspondería con un docente del tipo moderno, es decir, aquel que procura una revisión para mejorar el significado del conocimiento erróneo. Pero, no así, al utilizar las mismas técnicas, el mismo procedimiento de resolución, sin presentar situaciones registrales novedosas que permitan la utilización de otros registros que ayuden a ampliar los conceptos o vincularlos con otros conocimientos.

Los elementos de análisis pueden llevar a pensar que los errores de los alumnos se producirían por obstáculos de tipo didáctico, donde no se amplían las posibilidades representacionales propuestas por la docente. 


\section{ENTREVISTA NÚMERO 2}

De la entrevista a la segunda docente, surgió que utilizaba similares opciones didácticas que la anterior para explicar el tema, y vincular los conceptos con otros vistos con anterioridad, y aludió a que "no hay demasiadas estrategias para el tema".

- Hoy vamos a observar una clase sobre Expresiones Algebraicas Fraccionarias ¿De qué manera piensa desarrollar el tema? ¿Cómo iniciaría la clase?

- (...) No es un tema muy didáctico digamos, yo siempre hago la comparación con fracciones comunes y a partir de la suma de fracciones comunes doy la suma de expresiones algebraicas fraccionarias, a partir de la multiplicación, la simplificación de fracciones comunes hago una especie de comparación (RRN y transformación de conversión a RRA), pero no encuentro otra manera de enseñar este tema particularmente. Arranco dándoles un ejercicio de ejemplo y después les doy ejercicios de aplicación (RRA y transformación de tratamiento).

- ¿Qué estrategias didácticas consideras que colaboran al mejor entendimiento de las Expresiones Algebraicas Fraccionarias?

- No sé si hay estrategias didácticas para este tema, yo no las encuentro (...).

El tipo de estrategia mencionado por la docente permite que el alumno emplee sus conocimientos previos frente a situaciones novedosas a la vez que incluye a sus estructuras cognitivas las nuevas explicaciones (RRVL), y los nuevos contextos de aplicación. En la observación de clase se reconoció que la estrategia aplicada fue aceptada por los alumnos y llegó a interesarlos.

Cuando se preguntó sobre los recursos y actividades propuestas, contestó que comenzaba con explicación con ejemplos en el pizarrón y luego ejercitación, lo cual da cuenta de la utilización de RRVL y RRA, con operaciones de conversión entre ambos, y posterior tratamiento.

- ¿Qué actividades realizan los alumnos?

- (...) Ejercicios de aplicación, problemas cero, con este, cero.

- ¿Con el tema, o con este cuarto?

- (...) Con este tema, es como una continuación de polinomio, y es un tema que lo damos rapidito, no los engancha a ellos, yo particularmente no tengo muchas estrategias, acá siempre se da así el tema y se sigue con otra cosa (...). 
Se puede inferir que la docente realizaba tratamientos dentro del mismo registro, pero no diversificaba las conversiones, hizo constantemente aclaraciones y explicaciones orales que reforzaron el concepto algebraico, por lo cual se observó que otorga gran importancia al registro RRVL oral.

Al preguntar sobre los errores comunes de los alumnos, las respuestas también fueron similares al docente número 1, los alumnos no podrían aplicar temas vistos anteriormente, según respondió. Como posibles causas indicó que los alumnos no logran relacionar los temas, que repiten siempre los mismos errores y que son intrínsecos al Álgebra.

Por último, al preguntarle cómo trabaja sobre esas dificultades, al igual que en la entrevista número 1, la docente reconoció que aplica múltiples técnicas, por ejemplo, repasar lo dado antes de la evaluación (RRVL y RRA), dar ejercicios de aplicación más sencillos (transformación de tratamiento), o explicar el tema nuevamente (RRVL y RRA).

Esta diversidad en la revisión de lo erróneo podría conducir a caracterizar a ambos docentes consultados como del tipo moderno, y denotar con ello cierta disposición a fomentar ampliación de la actividad registral de representación semiótica (ver apartado 2.2).

\subsection{OBSERVACIÓN DE CLASE NÚMERO 1}

La profesora comenzó la clase corrigiendo ejercicios de la clase anterior realizando transformación de tratamiento. Esta actividad permitió recordar el tema y revisar posibles errores de resolución.

La profesora corrige el tercer ejercicio, pregunta al alumno cómo lo hizo, explica que si saca el -2 debe cambiar el signo. Corrige el ejercicio cuatro preguntando:

- ¿Qué podemos hacer? ¿Qué podemos hacer cuando se multiplican dos expresiones?

- Distributiva (contesta una alumna).

Utilizó los RRA al representar las ecuaciones, RRA, y RRVL (verbal conceptual), en el caso de las correcciones y explicaciones. La profesora involucró a los alumnos en la corrección de las actividades. No se aclararon o ampliaron conceptos matemáticos en la interacción. 
Un alumno preguntó sobre la propiedad distributiva, la profesora explicó paso a paso; en ese momento se trabajó dentro del RRVL y RRA, con utilización de algunos esquemas gráficos (flechas), vinculando el lenguaje con el procedimiento.

La profesora explica la propiedad distributiva escribiendo en el pizarrón la expresión factorial:

$$
(x+1)(x+3)=x^{2}+3 x+x+3
$$

Rápidamente borró lo que había escrito y los estudiantes se quejan debido a que no lograron copiar en sus carpetas.

Se pudo ver la operación de tratamiento dentro del RRA utilizada por la docente para pasar de los factores algebraicos, a la equivalencia con las sumas. No se diversificó la explicación en diferentes registros, ni se dio el tiempo suficiente para que los alumnos incorporasen a sus estructuras de pensamiento; al respecto, Duval destaca que la integración de los sistemas de representación a la estructura cognoscitiva es fundamental para comprender Matemática. Por otra parte, la conversión de un registro a otro es lo que le da significado a un objeto matemático. El RRVL en tal caso no participó en la explicación con supremacía respecto de otros registros.

Cuando la profesora comenzó a explicar el tema lo hizo como continuación del anterior, considerando que la suma y resta son operaciones que le suceden a la multiplicación y división. Escribió un ejemplo en el pizarrón donde se utilizó el RRA y RRVL para la explicación. Se realizó una comparación con fracciones numéricas mediante RRN. Se pudo observar conversión entre registros en la comparación, la cual implica aplicación de conocimientos previos de los alumnos, no siendo así en los casos de factoreo donde se observaron dificultades.

La profesora explicó que operar con expresiones fraccionarias requiere buscar común denominador, colocó el título en el pizarrón y escribió un ejemplo.

$$
\frac{x}{x-2}+\frac{x+2}{x-2}=\frac{x+x+2}{x-2}=\frac{2 x+2}{x-2}(\text { RRVL y RRA })
$$

(Pide que se fijen en el denominador: ¿Cómo son? Iguales, por lo tanto ¿Cuál sería el común denominador?) (RRVL)

Alumnos: X-2.

Profesora: (Desarrolla el ejercicio paso a paso en el pizarrón, indica que se puede aplicar el primer caso de factoreo, pero la expresión no se modifica, no se 
puede simplificar, por ese motivo lo dejan así (RRA y RRVL). Pide que copien mientras escribe otro ejercicio de un cuadernillo).

$$
\frac{x}{x-2}+\frac{3 x-1}{x^{2}-2 x}=\frac{x}{x-2}+\frac{3 x-1}{x(x-2)}=(\mathrm{RRA})
$$

Profesora: (escribe y pregunta), ¿Cómo son los denominadores? Distintos. En este caso se debe factorizar primero y luego encontrar el factor común. (RRVL y RRN)

Profesora: (Explica cómo obtenerlo con un ejemplo de números fraccionarios 3/2 + $2 / 3=/ 6$, recuerda que es el mayor de los números y pregunta cuál es en el ejemplo). (RRVL y RRN)

Alumnos: (no responden).

Profesora: (vuelve a preguntar) ¿Cuál se repite?

Alumnos: $X-2$, contestan.

Profesora: ¿Y qué más?

Resuelven y pregunta a la clase:

Profesora: ¿Qué más pueden aplicar? ¿El primer caso, el segundo, el tercero...?

Alumnos: Ruffini (contestan los alumnos).

Profesora: No me había enterado que Ruffini tenía un caso de factoreo...

Otro alumno contesta: "El tercer caso", la profesora trata de resolverlo, inmediatamente los alumnos se dan cuenta que no es posible, luego otro estudiante contesta: "Gauss".

Profesora: ¿Eso puede ser lo que pensaste como Ruffini?, le pregunta.

Pasa una alumna a resolverlo. En el pizarrón escribe $q=x^{2}$, la profesora aclara que es solo el coeficiente, la alumna lo corrige y aplica Regla de Ruffini correctamente, comprueba que no se puede factorizar. (RRA y RRVL)

La docente pretendió un tipo de interacción y de respuestas específicas no habiendo explicitado de manera verbal algunos requerimientos, lo cual puede interpretarse como error didáctico con origen en la formulación de las preguntas que la docente realizó a los alumnos (RRVL), por falta de transformaciones de registros. Las preguntas fueron poco específicas y carecían de conceptos distinguibles en un cierto tipo de RS transformable. 
Luego, pasó una alumna al pizarrón, la profesora indicó que lo que la estudiante escribía contenía un error en la diferenciación entre coeficiente y término, sin aclarar cuál era esa diferencia, por lo que podría persistir el mismo. Se pudo observar que la intervención del RRVL, utilizado para aclarar o ampliar conceptos fue lo que condujo al error. La profesora explicó un ejercicio de expresiones con distintos denominadores, pero no se ampliaron conceptos, ni se utilizaron otras estrategias para explicar. Continuó trabajando con RRA y RRVL no conceptual, lo cual se podría caracterizar como obstáculo didáctico donde no se amplían las opciones registrales.

La docente escribe otro ejercicio en el pizarrón y pregunta (RRA):

Profesora: ¿Cómo son los denominadores? ¿Cuál será el común denominador? ¿Qué dijimos que se hacía con los denominadores?

Alumnos: Se multiplican...

$$
\begin{aligned}
\frac{x}{x+1}-\frac{2}{x-1}=\frac{x(x-1)-2(x+1)}{(x+1)(x-1)} & =\frac{x^{2}-x-2 x-2}{(x+1)(x-1)} \\
& =\frac{x^{2}-3 x-2}{(x+1)(x-1)}
\end{aligned}
$$

(Resuelve el ejercicio con la participación de los alumnos).

Profesora: ¿Qué podemos hacer?

Alumnos: Simplificar.

Profesora: ¿Qué cosa?

Alumnos: Los paréntesis.

Profesora: No porque hay un signo menos (-), primero debe expresarse como producto. ¿Qué se puede hacer entonces?

Alumnos: Distributiva.

La docente comenzó interactuando con los alumnos para construir un nuevo concepto (suma algebraica) pero se vio interrumpida y no se logró el objetivo; se realizó mediante RRA sin explicaciones. Se observa tratamiento dentro de un registro desvinculado del contexto, como aplicación mecánica de las operaciones lo cual refuerza lo planteado por la profesora en la entrevista acerca de que los chicos no relacionan los temas.

Continuando con la clase la profesora escribe una suma algebraica en el pizarrón y pregunta (RRA): 
Profesora: ¿Cómo se llama cuando combinamos sumas y restas?

Alumno: Operaciones combinadas (contesta un alumno)

Alumno: Resma (contesta otro).

La profesora dice que están muy chistosos, que para la próxima clase deben averiguar cómo se dice.

$$
\begin{gathered}
\frac{1}{2 x-2}+\frac{2}{x^{2}-1}-\frac{x}{x+1}= \\
\frac{1}{2(x-1)}+\frac{2}{(x-1)(x+1)}-\frac{x}{x+1}=\frac{1(x+1)+2.2-2 x(x-1)}{2(x+1)(x-1)}
\end{gathered}
$$

Comienza a resolver el ejercicio en el pizarrón sin explicar (RRA y transformación de tratamiento), los alumnos averiguaron el común denominador correctamente. Un alumno pregunta cómo sacó ese 2 multiplicado por 2 (expresado como 2.2 en el numerador de la expresión racional última). La profesora explica que es como fracciones, se divide y multiplica. (RRVL)

Se tomaron decisiones didácticas del tipo tradicional, y poca implementación de RRVL sin desglose del contenido a través, por ejemplo, del diálogo y la participación activa de los alumnos.

\subsection{OBSERVACIÓN DE CLASE NÚMERO 2}

La docente comenzó la clase recordando lo dado en la clase anterior, con un ejemplo que explicó en el pizarrón. Utilizó RRVL, a medida que escribió, para señalar el tipo de expresión que representaría, sus características, y las operaciones que se incluían; y RRA, para simbolizar la tarea matemática, y el objeto expresión racional.

La profesora entra al aula y pide a un alumno que borre el pizarrón. Les recuerda que la clase anterior vieron suma y resta de expresiones algebraicas. Escribe un ejercicio en el pizarrón con expresiones algebraicas racionales de igual denominador y lo desarrolla a medida que va preguntando el procedimiento a los alumnos.

$$
\frac{1}{x}+\frac{2}{x}-\frac{5}{x}=\frac{1+2-5}{x}=\frac{-2}{x}
$$


Las intervenciones docentes fueron destinadas a propiciar el aprendizaje de nuevos conceptos. La aplicación reiterada de los conocimientos previos articulados a través de RRVL, recordándolos, otorgándoles nuevos significados o aplicaciones, permitió estructurar el nuevo conocimiento-objetivo de la clase y a la vez consolidar nociones anteriormente aprendidas.

El inicio de clase permitió interrelacionar los temas y organizar una serie de conceptos dentro de un mismo campo. Cuando se introdujo el tema Suma y Resta de Expresiones Algebraicas Racionales con distinto denominador se utilizó RRVL y RRA simultáneamente, haciendo aclaraciones mediante lenguaje matemático y cotidiano.

Realiza otro ejemplo con fracciones de distinto denominador y pregunta qué es lo común y lo no común en ellas:

$$
\frac{x}{2}+\frac{1}{x}+\frac{1}{1}=\frac{x^{2}+2+2 x}{2 x}
$$

A medida que escribe en la pizarra, comenta los pasos que realiza, y luego pregunta si se puede simplificar, recuerda que en la suma y en la resta solo es posible en forma vertical, es decir numerador y denominador de la misma fracción.

$$
\begin{gathered}
\frac{3}{x}+\frac{2 x}{x^{2}}=\frac{3}{x}+\frac{2}{x}=\frac{5}{x} \\
\frac{x}{2 x-6}-\frac{3}{x-3}=\frac{x}{2(x-3)}-\frac{3}{x-3}=\frac{x-6}{2(x-3)}
\end{gathered}
$$

En el momento en que los alumnos resolvían los ejercicios de manera autónoma, la profesora recordó en forma oral pasos procedimentales, involucró a los estudiantes en la resolución, aclaró ideas, conceptos y terminología.

Mientras los alumnos trabajan, la profesora recuerda los pasos: "Repetimos el denominador y sumamos los numeradores".

Una alumna pregunta si terminó ahí el ejercicio, la profesora explica:

Profesora: No se puede seguir resolviendo porque no son términos semejantes, si fueran todas $x^{2}$ o todas $x$ sí podrían sumar, por ejemplo, en este caso $(x-6)$ no queda $(-5 x)$ porque a las $x$ solo les puedo restar $x$. (RRVL y RRA) 


$$
\begin{gathered}
\text { c) } \frac{2 x-x^{3}}{x^{2}}-\frac{x(x+2)}{x^{2}}=\frac{2 x-x^{3}-x(x+2)}{x^{2}}=\frac{2 x-x^{3}-x^{2}-2 x}{x^{2}}=\frac{-x^{3}-x^{2}}{x^{2}} \\
\text { d) } \frac{3 x^{3}+1}{12 x^{2}}+\frac{5 x^{3}-1}{12 x^{2}}=\frac{3 x^{3}+1+5 x^{3}-1}{12 x^{2}}=\frac{8 x^{3}}{12 x^{2}}=\frac{2 x}{3}
\end{gathered}
$$

(La profesora indicó que $x^{3}=x . x . x$., y que $x^{2}=x . x$, y que por tanto se podía simplificar). Anteriormente había aclarado que en el ejercicio c) se repitió el denominador por ser iguales (señala) $2 x y-2 x$ se cancelan por ser positivo y negativo. (RRVL y RRA)

Los alumnos trabajaron con una fotocopia que contenía sintetizados los desarrollos de los casos de factoreo (registro algebraico); se observó que este recurso resultó útil ya que los estudiantes aplicaban los conocimientos con facilidad al nuevo tema y los vincularon de forma natural. El texto, un cuadro impreso distribuido en la clase, constaba de RRA, RRN, RRVL y RRGr, y símbolos aclaratorios y flechas.

Conformado por distintos registros sobre el mismo objeto matemático, implícitamente realizaba conversiones, lo que ayudó a los estudiantes a comparar las diferentes formas de expresar lo mismo y a conceptualizar, y permitió diversificar los modos de representación.

En el siguiente ejercicio los alumnos tenían dificultades para agrupar términos semejantes y diferenciar la suma de la multiplicación de expresiones algebraicas. La profesora utilizó tanto el RRA como RRVL para explicar, sin ampliar los conceptos.

Al dar el siguiente ejercicio, la docente explica con un ejemplo de fracciones numéricas, lo compara con el ejercicio anterior y pide a un alumno que lo resuelva, agrupa los términos semejantes, tres veces $x$ y dos veces $x$, lo resalta con color para simplificar. (RRN, RRVL, RRA, y transformaciones de conversión y tratamiento)

Algunos alumnos se paran a preguntar si hicieron bien el ejercicio, la profesora los corrige.

Profesora: Una $x$ más una $x$ son $2 x$ no $x^{2}$

(Los alumnos que están al frente se ayudan entre sí).

Profesora: Hay que buscar los que son parecidos, si tiene esto arriba $\left(x^{2}\right)$ no son. (RRVL y RRA) 
Se amplió la explicación y se dio mayor intervención RRVL para afianzar el aprendizaje. Algunas condiciones se explicitaron verbalmente a fin de lograr una mayor comprensión, no se realizó. Por ejemplo, lo referente a suma de términos con expresiones potenciales semejantes, o las propiedades de la suma y la potenciación.

La profesora vuelve a repetir la corrección para el resto de la clase Profesora: Si hago $x$ por $x$ es $x^{2}$, si sumo $x$ más $x$ son $2 x$. (RRA y RRVL) Resalta los términos semejantes en el ejercicio y resuelve (RRA):

$$
\frac{1+x}{x^{2}-1}+\frac{5 x^{2}+x}{x^{2}-1}-\frac{4 x^{2}}{x^{2}-1}=\frac{1+x+5 x^{2}+x-4 x^{2}}{x^{2}-1}=\frac{x^{2}+2 x+1}{x^{2}-1}
$$

Recuerda que en la suma y en la resta no se puede simplificar, en el ejercicio anterior sí porque el 8 multiplicaba a la $x^{3}$ y el 12 a la $x^{2}$ (RRVL y RRA).

Al explicar un nuevo ejercicio se utilizó el RRVL oral y el RRA para referenciar los procedimientos a seguir. La profesora interactuó con los alumnos preguntando específicamente aspectos del contenido matemático, realizó transposición didáctica introduciendo terminología matemática, y reafirmó los conceptos en el lenguaje cotidiano.

Continúa con ejercicios de distinto denominador, explica que primero deben factorizar (RRVL y RRA con transformaciones de tratamiento):

Profesora: Como primer paso traten de factorizar lo que puedan, en la primera expresión ¿Qué caso puedo aplicar?

Alumno: En el numerador imposible porque tengo 1, ¿en el denominador?

Profesora: Factor común.

Alumno: ¿Cuál?

Profesora: Dos.

Explica como extraer el factor común y señala con una llave lo realizado; de la misma manera lo hace para el segundo término (RRVL, RRA, y RGr):

$$
\underbrace{\frac{1}{2 x-2}}+\underbrace{\frac{2}{x^{2}-1}}-\frac{x}{x+1}=
$$

Indica que en el primer denominador se aplica factor común, que en el segundo corresponde diferencia de cuadrados, y que en el tercer denominador no se puede 
porque es suma. Mediante las expresiones "F.C" y, "D.C" deja escrito debajo de cada llave dibujada el tipo de factoreo. (RRVL)

Seguidamente aplica factoreo y lo anota en el pizarrón. (RRA)

$$
(2 x-2)=2(x-1) \quad x^{2}-1=(x+1)(x-1)
$$

Para el segundo denominador explica la equivalencia de las bases cuadráticas a las expresiones que a continuación escribió, y vinculó a la aplicación de la diferencia de cuadrados. (RRVL y RRA)

$$
\sqrt{x^{2}}=x \quad \sqrt{1}=1
$$

La profesora asoció cada base al término correspondiente de la diferencia de cuadrados mediante flechas. (RRVL y RGr)

Profesora: ¿Qué puedo aplicar en la segunda expresión fraccionaria algebraica?, en el 2 obviamente que no, ¿Y en el ( $\left.x^{2}-1\right)$ ? (RRVL y RRA)

Alumnos: Diferencia de cuadrados

Profesora: Si son dos términos cuadráticos y es una resta aplico diferencia de cuadrados, tomo las dos raíces extraídas y lo escribo como la suma por la diferencia. (RRVL)

Se observó nuevamente que la docente introdujo terminología matemática, y reafirmó los conceptos en el lenguaje verbal natural.

Continúa con el tercer término preguntando a los alumnos:

Profesora: ¿Puedo simplificar algo?

Alumnos: Sí, la x.

Profesora: No, porque está sumando con el uno.

Escribe y nombra los términos factorizados, luego resuelve el ejercicio recordando los pasos (RRA y RRVL):

$$
\frac{1}{2(x-1)}+\frac{2}{(x+1)(x-1)}-\frac{x}{x+1}=\frac{1(x+1)+4-2 x(x-1)}{2(x+1)(x-1)}
$$

Profesora: Cuando tienen distinto denominador, factorizo y escribo cada polinomio por el polinomio obtenido al factorizar. Sacamos común denominador, ¿cuál va a ser el común denominador? Lo común y lo no común... (RRVL).

Alumnos: $2(x-1)$ 
Profesora: El 2 es no común por $(x-1)$, acá tengo $(x-1)$ por $(x+1),(x-1)$ ya lo puse entonces pongo $(x+1)$ y el $(x+1)$ también se repite así que ese va a ser el común denominador. Lo común y lo no común. (RRA y RRVL)

Comienza a dividir con la participación de los alumnos:

Profesora: Todo esto dividido, me queda lo no común, por uno queda igual, más todo esto dividido, esto me queda... (RRA y RRVL (lengua natural)).

Cuando los estudiantes dudaron de la explicación, la profesora revisó sintéticamente los pasos y explicó la dificultad utilizando RRVL y RRA.

Una alumna interrumpe diciendo que no entiende como hace la división, la profesora explica la división como una fracción, la escribe por separado (RRVL, RRA, RRN):

$$
\frac{2(x-1)(x+1)}{2(x-1)}=x+1
$$

Profesora: Vamos a escribir la división como una fracción porque la raya de fracción indica división. ¿Puedo simplificar algo? (RRVL)

Alumnos: Sí, el 2( $x-1)$.

Profesora: Me quedaría el $(x+1)$, entonces siempre que divido algo con otra cosa el resultado de esa división es lo que no es común, porque 2 dividido 2 es $1,(x-1)$ dividido $(x-1)$ también es uno y $(x+1)$ es lo que me sobra. (RRA, RRVL)

Continúa con la explicación del ejercicio y termina de resolverlo.

$$
\begin{gathered}
\frac{1}{2(x-1)}+\frac{2}{(x+1)(x-1)}-\frac{x}{x+1}= \\
\frac{1(x+1)+4-2 x(x-1)}{2(x+1)(x-1)}=\frac{x+1+4-2 x^{2}+2 x}{2(x+1)(x-1)}=\frac{-2 x^{2}+3 x+5}{2(x+1)(x-1)}
\end{gathered}
$$

En ese paso, que no fue representado algebraicamente, pudo conducir a un error al no realizar la conversión de registros. Luego, las decisiones didácticas se destinaron a proporcionar una técnica diferente y se intentó explicar de nuevo el procedimiento, lo cual facilitó la comprensión. 


\section{DISCUSIÓN}

La TRRS, permite afirmar que, en Enseñanza de la Matemática, la actividad utilizada para el tratamiento y la conversión entre diferentes sistemas representacionales tiene gran importancia. Las transformaciones incorporan las bases de la conceptualización y la comprensión matemática, con el valor adicional de que los contextos de representación utilizados son semióticos; así el procesamiento matemático implica siempre una transformación de representaciones.

El contenido de cada representación no depende únicamente de los objetos representados, sino también del tipo de $R S$ utilizado con intenciones didácticas. Se observó, en ambos grupos, que utilizaron básicamente RRA y RRVL de manera prevalente, y otras modalidades en la representación semiótica como lo es la gráfica, con flechas, y llaves, pero, al igual que la numérica, se presentan con menor frecuencia. En el primero de ellos, el RRVL adoptó formas verbales cotidianas con escasos elementos de contenidos terminológicos específicos, y conceptuales. En el segundo, se combinaron ambos registros, con una mayor presencia de elementos lingüísticos matemáticos, lo que permitió tanto conversiones como tratamiento de los mismos de manera eficiente.

Se observó que en el primer grupo hubo poca interacción por parte de los alumnos, estos tenían dificultades en la resolución de los ejercicios, no podían vincularlos con otros temas y las intervenciones de la docente eran correctivas, pero no se ampliaban conceptos matemáticos. En el segundo grupo, se observó participación de los alumnos espontáneamente, guiada por la profesora mediante preguntas y explicaciones que se formularon frecuentemente durante el desarrollo de la clase. En el caso de errores cometidos por los estudiantes, estos eran aclarados en vinculación con otros conceptos matemáticos, por lo que se permitió una retroalimentación de articulación entre el RRA y RRVL. La presencia de más formatos representacionales (verbal, algebraico, y gráfico, como es el caso del apunte de ayuda memoria escrito que entregó la docente), y los modos de relacionar las diferentes maneras de representar los mismos objetos, contribuyó a la conceptualización y comprensión por parte de los estudiantes.

Se puede afirmar, en coincidencia con la descripción de la TRRS, que la enseñanza de un determinado objeto matemático debe producirse apelando a distintos sistemas de RS, puesto que el primer paso para la comprensión es que se produzca una coordinación interna entre los diferentes sistemas de representación disponibles, como se ha señalado en el marco teórico. 
Cabría la posibilidad de plantear la idea que indicaría que, si el alumno es conducido a conocer solamente un tipo de registro, difícilmente lo comprendería y aplicaría a otros contextos o registros. La vinculación con conocimientos previos y su correcta aplicación deberían llevarnos a la pregunta respecto de si ello requiere de la guía docente, con constantes intervenciones aclaratorias, recordatorias o ampliatorias del concepto o campo de aplicación, lo cual equivaldría a una expansión máxima del RRVL en su función semiótica articular de otros registros.

Para la Enseñanza de las Expresiones Algebraicas Racionales, sería indispensable que los docentes trabajen con aclaraciones en los pasos de procedimientos, escritura algebraica específica, tanto en RRVL (orales o escritos), como RRA. Lo antes dicho se infiere del análisis de las prácticas de las profesoras entrevistadas. De esta manera se posibilita que objetos del sistema antiguo de signos de los estudiantes, es decir, conocimientos constituidos en prerrequisitos de aprendizaje, no representen dificultades, sino que, por el contrario, posibiliten adquisición de nuevos contenidos de aprendizaje. No se debería apresurar el aprendizaje de un nuevo objeto matemático ya que los tiempos de aplicación de los registros, su tratamiento y conversión, en expansión lingüística, servirían para incorporar el nuevo concepto o conocimiento a estructuras cognitivas.

En ambos grupos de investigación, de acuerdo a los datos presentados, se observaron pocas transformaciones, del tipo conversión; sí hubo tratamientos, en el caso del RRA, tanto por parte de los alumnos como de las docentes. Los profesores no deben olvidar las restricciones de los RS acorde a la abstracción y generalidad de los conceptos didácticos y matemáticos. En particular, ante el tema Expresiones Algebraicas Racionales fue conveniente comenzar con ejemplos sencillos, para luego aplicar procedimientos y técnicas vistas con anterioridad como, por ejemplo, los casos de factoreo. Este aspecto fue planteado por la segunda docente con mayor detalle, quien además vinculó conceptos con un cuadro que contenía el mismo objeto matemático expresado en diferentes RS.

El error didáctico y las dificultades de aprendizaje significarían, a la luz de los elementos considerados de la TRRS, deficiencias en las elecciones de registros que los docentes efectúan y en las actividades matemáticas registrales que los estudiantes desarrollan para acrecentar la potencialidad representacional en la construcción de los objetos y conceptos matemáticos. De tal manera, un profesor convencional, para subsanar el error, no admitiría, contrariamente al posicionamiento de un profesor moderno, la necesidad de introducir mejoras en las secuencias y acciones registrales mediante las cuales ellos enseñan y los alumnos aprenden. 


\section{CONCLUSIÓN}

De lo analizado resultaría particularmente importante fundamentar la enseñanza de expresiones algebraicas racionales teniendo en cuenta el ordenamiento de los distintos RS de la clase. Se ha visto que es preciso propiciar la expansión del RRVL, es decir realizar el máximo posible de intervenciones orales o escritas considerando que la utilización del mismo podría ser, en el enfoque conceptual de la TRRS, el medio no solo de comunicación y ampliación de conocimientos sino también de incorporación cognitiva de conocimientos matemáticos.

Presentar en una jerarquía registral adecuada en un ordenamiento didáctico particular de diferentes RS implicaría expresar por ejemplo de forma oral ese contenido matemático escolar a enseñar (RRVL disciplinar), para traducir lo dicho en términos coloquiales luego en lenguaje matemático (RRA). De acuerdo a lo observado, esta secuencia de registros admitiría una organización más eficaz del conocimiento matemático (lo cual pudo verse en el segundo caso analizado), facilitando la representación de situaciones complejas de modo preciso, formal y comprensible en esa articulación registral.

Resultaría, de acuerdo a deducciones obtenidas en la investigación realizada (aceptando que los resultados de la misma no son generalizables), recomendable que, por ejemplo, mientras un docente enseña tales contenidos, expanda coloquialmente mediante palabras claves o aclaratorias aquello que escribe en forma algebraica en el pizarrón, o en el soporte compartido por la clase, y genere así un entorno lingüístico extendido para definir y verificar los sentidos que se asignan a tales definiciones. Ampliar dicho registro serviría no solo de ayuda memoria a los estudiantes sino también de recuperación de significados y antecedentes cognitivos para continuar aprendiendo.

En ambos grupos de observaciones se propuso desarrollar el mismo tema con idénticas técnicas e idénticos registros. En la primera clase observada se presentó la actividad matemática con predominio de RRA, mediante un ejemplo; después una breve explicación empleando el registro lingüístico y, por último, se resolvió el ejercicio propuesto con nuevo predominio de RRA. En un primer momento los estudiantes no comprendieron la situación y luego no comprendieron los procedimientos aplicados para la resolución. También se evidenció que faltaron elementos discursivos al representar los objetos matemáticos y las operaciones con ellos. En la segunda clase observada, la docente comenzó recordando la clase anterior de forma oral, explicó ejemplos sencillos de manera algebraica, asistiendo permanentemente la enseñanza mediante RRVL, plasmó los pasos realizados tanto en 
RRA como en RRVL en el pizarrón y luego volvió a recordar todos los procedimientos de forma oral, lo que llevó a un mejor entendimiento por parte de los alumnos y a una interacción en la construcción del conocimiento.

Se concluye que es posible que, el ordenamiento en que se presentaron distintos RS en la enseñanza de expresiones algebraicas racionales, influiría en la mejora de los aprendizajes de tales contenidos (aun trabajando solamente con dos sistemas de representación y su combinación), comenzando siempre por el RRVL conceptual, seguidamente del RRA, con una constante coordinación entre RRVL y RRA, mientras se procura aumentar al máximo el número de explicaciones (RRVL) al implementar diferentes registros. Tal articulación favoreció la conversión y el proceso de construcción didáctica del objeto matemático enseñado.

No fue posible localizar una gran diversidad de registros, y ello quizás responda especialmente al tipo de contenido. Por tanto, tampoco fue posible poner en evidencia de qué manera se desarrollaría la enseñanza del contenido si se utilizara una mayor diversidad de RS.

Se sugiere investigar la enseñanza de expresiones algebraicas racionales, su tratamiento dentro de un mismo registro, y conversiones de RS, donde se otorgue mayor importancia a la producción de diferentes formatos de representación fortalecidos por la presencia representacional de RRVL en su calidad semiótico-articular en acciones de docentes al enseñar y de estudiantes al aprender.

En tales análisis el RRVL, debería estar presente a lo largo de todo el desarrollo del contenido para trabajar a partir de la hipótesis de que el RRVL posibilita ampliar, completar y articular conocimientos como nexo vinculante de los demás registros, puesto que, como se dijo, el trabajo con objetos abstractos del pensamiento matemático depende del lenguaje y de otros sistemas de representación semiótica, cuya principal dificultad sería la de adquirir la habilidad para cambiar de un RS a otro.

Desde este trabajo se obtuvo información acerca de la vinculación entre RS y errores didácticos. Sería posible vincular estos últimos a vacancias de RRVL en la brecha entre los diferentes sistemas de representación utilizados para la enseñanza de la matemática escolar. Ello requeriría ser enfatizado en los objetivos de investigaciones posteriores que mejor lo confirmen.

Se puede concluir parcialmente que, los errores didácticos (decisiones y opciones didácticas), tendrían una base causal en la disminución de la presencia de RRVL como vínculo inter-representacional, lo cual acordaría también, en que la expansión de las posibilidades registrales previstas por las docentes aumentaría el potencial de las propuestas didácticas para su comprensión. 
Se recomienda continuar en dichas líneas de investigación para colaborar con el desarrollo de una didáctica específica sobre determinados contenidos curriculares matemáticos, en general, y en particular de la Didáctica del Álgebra, en los cuales se puedan incorporar diferentes RS y secuenciar un orden que favorezca el aprendizaje, por ejemplo, en programación lineal, ecuaciones, y funciones, donde se podría proponer una actividad con otros tipos de RS además del RRA y RRVL, como lo serían el RRGr y el RRT.

\section{REFERENCIAS}

Aponte Rodríguez, H. (2016). Coordinación de Registros Semióticos en la Presentación de la Periodicidad, el Acotamiento y la Conversión de Unidades de las Funciones Trigonométricas Seno y Coseno. Análisis de Texto. (Trabajo de Grado. Universidad del Valle). http://funes.uniandes.edu.co/11036/

Blumer, H. (1982). El Interaccionismo Simbólico: perspectiva y método. Hora S. A.

Bohorquez, H., Franchi Boscán, L., Hernández, A. I., Salcedo, S., y Morán, R. (2009). La concepción de la simetría en estudiantes como un obstáculo epistemológico para el aprendizaje de la geometría. Revista Educere, 13(45), 165-198.

Brousseau, G. (1983). Los obstáculos epistemológicos y los problemas en matemáticas. Recherches en Didactique des Mathematics.

Brousseau, G. (1983). Obstacles épistémologiques et les problémes en mathématiques. Recherches en Didactique des Mathématiques, 4(2), 165-198

D’Amore, B. (2004). Epistemología, didáctica de la matemática y prácticas de enseñanza. Revista de la ASOVEMAT, 17(1), 1-20.

D’Amore, B., y Godino, J. D. (2007). El enfoque ontosemiótico como un desarrollo de la teoría antropológica en didáctica de la matemática. Revista latinoamericana de investigación en matemática educativa, 10(2), 191-218. http://www.scielo.org.mx/ scielo.php?script=sci_arttext\&pid=S1665-24362007000200002\&lng=es\&tlng=es.

D’Amore, B., y Godino, J. (2007). El Enfoque Ontosemiótico como un Desarrollo de la Teoría Antropológica en Didáctica de la Matemática. Revista latinoamericana de investigación en matemática educativa, 10(2), 191-218. http://www.scielo.org.mx/ scielo.php?script=sci_arttext\&pid=S1665-24362007000200002\&lng=es\&tlng=es.

D’Amore, B. (2009). Conceptualización, registros de representaciones semióticas y noéticas: Interacciones constructivistas en el aprendizaje de los conceptos matemáticos e hipótesis sobre algunos factores que inhiben la devolución. Revista Científica. (11), 150-164. https://doi.org/10.14483/23448350.419 
Duval, R. (1999). Semiosis y pensamiento humano: registros semióticos y aprendizajes intelectuales. Grupo de Educación Matemática. Universidad del Valle.

Duval, R. (2004). Semiosis y pensamiento humano: registros semióticos y aprendizajes intelectuales. Instituto de Educación Pedagógica. Universidad del Valle.

Duval, R. (2006). Un tema crucial en la educación matemática: La habilidad para cambiar el registro de representación. Gaceta de la Real Sociedad Matematica Española, 9(1), 143-168.

Duval, R. (2017). Semiosis y pensamiento humano: registros semióticos y aprendizajes intelectuales. Traducción al español por Myriam Vega Restrepo, de Sémiosis et pensée humaine. Registres sémiotiques et apprentissages intellectuels. 2ª Edición. Programa Editorial Universidad del Valle.

Duval, R., y Sáenz-Ludlow, A. (2016). Comprensión y aprendizaje en matemáticas: Perspectivas semióticas seleccionadas. Énfasis. Universidad del Valle. Universidad Distrital Francisco José de Caldas. Universidad Pedagógica Nacional. Doctorado Interinstitucional en Educación.

Engler, A., Gregorini, M. I., Müller, D., Vrancken, S., y Hecklein, M. (2015). Los errores en el aprendizaje de matemática. Facultad de Ciencias Agrarias. Universidad Nacional del Litoral.

Gazzola, M. P., Llanos, V., y Otero, M. (2011). Funciones racionales en la Secundaria. Primeros resultados de una actividad de estudio e investigación. Tandil. Actas del I CIECyM, II ENEM.

Oviedo, L. M., Kanashiro, A. M., Bnzaquen, M., y Gorrochategui, M. (2011). Los registros semióticos de representación en matemática. Revista Aula Universitaria, 1(13), 29-36. https://doi.org/10.14409/au.v1i13.4112

Rico, L. (1997). Reivindicación del error en el aprendizaje de las matemática. Revista Épsilon, 38, 185-198.

Ramírez Sandoval, O., Romero Félix, C. F., y Oktaç, A. (2013). Coordinación de registros semióticos y las transformaciones en el plano. I OCEMACYC.

Sara, A., Scardigli, M., Pustilnik, I., Cittadini, G., y Pano, C. O. (2006). Errores recurrentes en el aprendizaje del álgebra lineal y registros de representación semiótica. XIII Jornadas de Investigación y Segundo Encuentro de Investigadores en Psicología del Mercosur. Facultad de Psicología - Universidad de Buenos Aires. https://www.aacademica.org/000-039/319

EUGENIO ARIEL VALIERO

Dirección: Colón N.3 326, CP 2840 (Gualeguya, Entre Ríos, Argentina)

Teléfono: $\quad$ +54 93444 57-1226. 